\title{
La convergencia entre las tasas de desempleo europeas
}

\author{
Raquel Llorente Heras ${ }^{1}$ \\ SERVILAB y Universidad de Alcalá
}

Recibido el 31 de enero de 2005; aceptado el 9 de junio de 2005

Received 31 January 2005; accepted 9 June 2005

\section{Resumen:}

Ante el reciente proceso de convergencia macroeconómica desarrollado dentro de la Unión Europea (UE) acorde con la implementación de los criterios de Maastrich y en un entorno de integración económica, cabe preguntarse que sucede en los mercado de trabajo. El presente artículo estudia la evolución del desempleo dentro de los países de la UE con el ánimo de demostrar la existencia de un leve pero cierto proceso de convergencia entre las tasas de desempleo europeas. Para ello se aplica al tema del desempleo dos medidas de convergencia propias de la Teoría del crecimiento, la $\beta$-convergencia y la $\sigma$-convergencia, y se desarrolla un ejercicio de cointegración para comprobar cuál es la relación existente entre las tasas de desempleo europeas a largo plazo.

Palabras clave: desempleo, Unión Europea, $\beta$-convergencia, $\sigma$-convergencia, cointegración. Clasificación JEL: J60, F02, O11

\begin{abstract}
:
Given the recent process of macroeconomic convergence and economic integration of the European Union fostered by the implementation of the Maastrich criteria, the situation of the labour market in this regard needs to be investigated. This article studies the evolution of unemployment rates in the EU countries to determine whether they tend to converge. Two measures of convergence widely used in growth theory, namely betaconvergence and sigma-convergence, are calculated, leading to the conclusion that a slight, though significant, process of convergence of unemployment rates does exist. Also a cointegration test is run in order to study the long-term relationship among European unemployment rates.
\end{abstract}

Keywords: unemployment, European Union, $\beta$-convergence, $\sigma$-convergence, cointegration. JEL Classification: J60, F02, O11

1 Dirección para correspondencia: raquel.llorente@uah.es Los hallazgos de este artículo provienen de mi tesis doctoral. Agradezco a mi tutora de tesis $\mathrm{D}^{\mathrm{a}}$ Inmaculada Cebrián López su constante apoyo sin el cual esta investigación jamás hubiera visto la luz. No obstante, todos los errores u omisiones cometidos en este artículo son totalmente atribuibles al autor.

(C) Revista de Economía Laboral 


\section{Introducción.}

El presente artículo analiza si existe un acercamiento entre las tasas de desempleo de los países europeos, de modo que dentro del mercado de trabajo pueda existir una cierta convergencia reflejo de la convergencia macroeconómica alcanzada dentro del proceso de integración europea. La integración macroeconómica que se esta produciendo dentro de la Unión Europea (UE) es un proceso histórico con importantes efectos dentro del mercado de trabajo entre los cuales podría estar el acercamiento o aproximación de las diferentes tasas de desempleo europeas.

La estructura de este artículo es la siguiente: en primer lugar, se realiza una breve revisión teórica sobre cuáles son las posibilidades de que exista un proceso de convergencia en términos del desempleo. En segundo lugar, se establece como se va a medir la convergencia en términos de desempleo determinando que se aplicará al tema del desempleo los instrumentos de medida de la teoría del crecimiento (la $\beta$-convergencia y la $\sigma$ convergencia). En el apartado número 3 se realiza una aproximación a la situación actual de la convergencia europea en relación con el desempleo, para a continuación, en el apartado 4 realizar una completa medición de dicha convergencia a través de las pautas establecidas en los apartados anteriores. De forma complementaria, en el apartado 5, se desarrolla un ejercicio de cointegración donde se analiza cuál es la relación existente entre las tasas de desempleo europeas a largo plazo intentando validar si la de convergencia es un proceso firme y duradero. Por último, las conclusiones recogen los principales resultados alcanzados. Los análisis previos es se han desarrollado utilizado los datos de la Labour Force Survey (OCDE), base de datos que cuenta con series temporales sobre desempleo amplias, completas y comparables para todos los países de la UE.

\section{El proceso de convergencia entre las tasas de desempleo algunos aspectos relevantes desde el punto de vista teórico.}

¿Por qué puede producirse un proceso de convergencia entre las tasas de desempleo de los países europeos? ¿Qué factores pueden desencadenar un acercamiento entre las tasas de desempleo europeas? Hoy en día, no existen demasiadas teorías dedicadas a analizar de forma directa y completa estas cuestiones. Sin embargo, del estudio de los principales modelos sobre el mercado de trabajo puede extraerse una serie 
de casos o posibilidades donde la convergencia entre las tasas de desempleo es posible.

En primer lugar, desde un enfoque ortodoxo del mercado de trabajo $^{2}$ podemos establecer que la convergencia entre las tasas de desempleo de las naciones europeas esta determinada por las siguientes cuestiones:

La movilidad de los trabajadores que permite alcanzar el equilibrio de los diferentes mercados de trabajo nacionales. La existencia o la concentración del desempleo en determinados países puede compensarse con las vacantes existentes en países vecinos. Las migraciones se mueven comparando el nivel de desempleo de origen y de destino como indicador de la probabilidad de encontrar empleo (Antolín, 1995) y de este modo se compensan los desequilibrios del mercado de trabajo. Al reducir la tasa de desempleo de aquellos países con excedente de mano de obra se desencadena un cierto proceso de convergencia ${ }^{3}$.

El proceso de integración europea esta provocando un acercamiento de las instituciones que gobiernan los diferentes mercados de trabajo europeos. El desarrollo de la Estrategia Europea de Empleo (EEE) sobre el mercado de trabajo ha provocado la implementación de una serie de políticas comunes a todos los países miembros de la UE. Si consideramos que el desempleo es uno de los principales resultados que nacen del hecho de que el mercado de trabajo funcione como una institución social (Solow, 1979, 1980 y 1992); la aproximación de las instituciones que gobiernan el mercado de trabajo europeo puede desencadenar una aproximación de las tasas de desempleo europeas y, por tanto, un proceso de convergencia. Además, gran parte de la política europea en materia laboral se encamina a la reducción generalizada del desempleo, a la eliminación del desempleo estructural, a la reducción de las disparidades regionales y nacionales en materia de desempleo y a la eliminación de la concentración del desempleo en determinados colectivos poblacionales como son los jóvenes y las mujeres. Todo ello favorece la posible existencia de convergencia a nivel macroeconómico entre las tasas de desempleo europeas. En este sentido, Bentolila y Jimeno (2003) demuestran que la reducción del desempleo español tiene como una de sus causas la buena actuación desarrollada por las instituciones laborales.

\footnotetext{
2 Una completa descripción sobre este tipo de modelo puede encontrarse en cualquier manual de economía laboral. Véase, por ejemplo, MacConnell, et al (2003).

${ }^{3} \mathrm{El}$ desequilibrio de los mercados de trabajo europeos también podría venir de la mano de una reducción de las disparidades en los salarios. Sin embargo, estudios de la Comisión Europea (2001) y la tesis doctoral de Llorente (2005) demuestran la falta de convergencia entre los salarios europeos imposibilitando desde el punto de vista empírico esta posible vía de equilibrio.
} 
Sin embargo, dentro del enfoque ortodoxo del mercado de trabajo se da por supuesto una serie de condicionantes muy difíciles de hallar en la realidad tales como: la existencia de información perfecta sobre la situación laboral de los mercados de trabajo locales y vecinos, la coincidencia de estructuras sectoriales similares o complementarias que permitan encontrar un empleo adecuado o la homogeneización cualificativa y educativa de la mano de obra; factores que pueden limitar o reducir la convergencia de las tasas de desempleo.

No obstante, existen otros modelos sobre el mercado de trabajo bajo los cuales también es posible la existencia de una cierta convergencia en términos del desempleo. Dentro del modelo neoclásico del mercado de trabajo ${ }^{4}$ la convergencia entre las tasas de desempleo es posible gracias a la movilidad de los trabajadores que permite alcanzar el equilibrio de los mercados a nivel internacional. En este contexto, la actuación de las instituciones europeas también puede desencadenar un proceso de convergencia entre las tasas de desempleo europeas a través de la flexibilización y reducción de los salarios.

Asimismo, el desempleo puede estar causado por una demanda deficiente o insuficiente originada por un bajo nivel en la actividad económica a nivel agregado, es lo que comúnmente se conoce como desempleo keynesiano. En este caso también la convergencia entre las tasas de desempleo es posible a través de una reestructuración sectorial, de una movilización de los consumidores o de la innovación tecnológica, factores que en última instancia reactiven la demanda agregada $\mathrm{y}$ permitan una reducción generalizada del desempleo.

Aunque, estrictamente no se traten de modelos sobre el mercado de trabajo los modelos sobre el intercambio de bienes también pueden aportar una serie de argumentos a favor de la existencia de una cierta convergencia en términos del desempleo. Los modelos sobre el intercambio de bienes basados en las teorías de Ricardo (1817), tal como el modelo Heckscher-Ohlin, establecen que la ventaja del intercambio comercial se basa en la comercialización de un cierto trabajo incorporado en los bienes y servicios a través de la especialización productiva. La existencia del desempleo dentro de estos modelos se compensa bien a través de la movilidad de la mano de obra o a través de variaciones en la productividad de los trabajadores que provoca un aumento del comercio. En el primer caso, se produce una convergencia entre las tasas de desempleo gracias de nuevo a la migración.

\footnotetext{
${ }^{4}$ El modelo neoclásico sobre el mercado de trabajo determina, en líneas generales, que el desempleo es el resultado de la existencia de salarios elevados inflexibles a la baja Véase, de nuevo, McConnell, et al (2003).
} 
En definitiva, desde el marco teórico actual existen modelos y teorías que apoyan la posibilidad de que exista un cierto proceso de convergencia entre las tasas de desempleo. El objetivo de este artículo es demostrar si existe o no dicha convergencia entre las tasas de desempleo de los países europeos tal como se ha establecido previamente.

En la actualidad, la convergencia entre las tasas de desempleo ya han sido analizada solo que fundamentalmente desde una perspectiva regional (Amstrong y Taylor, 2000) (Quah, 1995). Blanchard y Katz, en 1992 determinaron que el movimiento de los trabajadores entre las diferentes regiones contribuye a la reducción del desempleo y a la convergencia de los mercados laborales locales. Dentro de este ámbito, otro trabajo muy interesante a destacar sería el desarrollado por Decresin y Fatás (1995) sobre la evolución de las disparidades regionales en Europa. Recientemente, Bayer y Juessen (2004) han demostrado la existencia de una débil convergencia entre las tasas de desempleo de las regiones alemanas bajo la utilización de test univariantes más potentes y adecuados que las medidas utilizadas hasta el momento para la medición de las diferencias regionales. En la mayoría de estos trabajos siguiendo a Bernard y Durlauf (1996) se estima que entre las tasas de desempleo regionales existe una convergencia en el largo plazo hacia un valor constante, de modo que:

$$
\forall: \lim _{k \rightarrow \infty} E\left(u_{i, t+k}-u_{j, t+k} I_{t}\right)=\text { constante }
$$

donde $u_{i}$ y $u_{j}$ refleja la convergencia entre la tasa de desempleo y la tasa en el equilibrio.

De acuerdo con la formulación anterior, una de las formas de contrastar la existencia de convergencia consiste en comprobar si las series temporales de las tasas de desempleo presentan algún tipo de acercamiento o convergencia entre sus tendencias a largo plazo, es decir, desarrollar un análisis de cointegración. En la parte final de este artículo, de acuerdo con las ideas previas se desarrolla un análisis de cointegración sobre las tasas de desempleo europeas.

Por último establecer, que a pesar del interés de estas cuestiones los estudios sobre la existencia de convergencia a nivel nacional resultan hoy en día bastante escasos. No obstante, entre otros puede destacarse el trabajo realizado por Van der Horst (2003) donde se demuestra lo débil del equilibrio existente entre las tasas de desempleo europeas. 


\section{La convergencia a largo plazo y su aplicación al tema del desempleo.}

Definir la convergencia desde un punto de vista macroeconómico en términos de desempleo resulta un tanto complejo. Para definir la convergencia a nivel macroeconómico, en este artículo se aplica al tema del desempleo los conceptos de convergencia originarios de la Teoría del crecimiento. Tradicionalmente, el estudio de la convergencia se ha realizado sobre la evolución del PIB o la renta. En este ámbito destacan entre otros muchos estudios los trabajos realizados por Quah (1993) Sala i Martín (1990 y 1994) De la Fuente (1996 y 1998) y Sachs y Warner (1995). Aplicando las Teorías del crecimiento recogidas en estos artículos al tema del desempleo, se puede calcular la convergencia entre las tasas de desempleo a través de dos aproximaciones, la estimación de la $\beta$ convergencia y la $\sigma$ convergencia. Estos conceptos que han resultado fundamentales en los estudios sobre la convergencia en términos de la renta, en la actualidad, están siendo utilizados para medir nuevos tipos de convergencia ofreciendo en algunos casos buenos resultados. Ejemplo de ello, es su aplicación al seguimiento de los precios y la inflación de Gaulier y Haller (2000) o incluso el estudio sobre la productividad laboral de Mulder y de Groot (2003).

\subsection{La $\beta$-convergencia y su aplicación al desempleo.}

El concepto de $\beta$-convergencia, originario de la teoría del crecimiento $^{5}$, nace como un instrumento para estimar el acercamiento o aproximación de las economías en términos de las tasas de crecimiento del PIB o de la renta (Barro, 1997). La $\beta$-convergencia supone que la tasa de crecimiento es una función negativa del nivel de renta del periodo anterior. Esto conlleva que los países más pobres, es decir, con un nivel de renta inicial menor, deben crecer a un ritmo superior para alcanzar a los países más ricos o al menos a la media del conjunto produciéndose un proceso de acercamiento o caching up. Si adaptamos la expresión matemática sobre la convergencia en términos de renta establecida por Xavier Sala-i-Martin (1994) al tema del desempleo el resultado sería el siguiente:

\footnotetext{
${ }^{5}$ Los fundamentos de la teoría del crecimiento pueden consultarse con detalle en el trabajo desarrollado por Solow (1956).
} 


$$
\log \left(u_{i, t}\right)-\log \left(u_{i, t-1}\right)=a-\beta \log \left(u_{i, t-1}\right)+\varepsilon_{i t}
$$

Esta ecuación mide la diferencia entre las tasas de crecimiento del desempleo en relación con el nivel desempleo inicial; el término $\varepsilon_{i t}$ es la perturbación con una distribución $\left(O ; \sigma^{2}\right)$ independiente del tiempo e idénticamente distribuida, el coeficiente $\beta$ que es una constante positiva entre cero y uno que indica la intensidad de la convergencia. La ecuación (2) puede simplificarse y escribirse del siguiente modo:

$$
\log \left(u_{i, t}\right)=a^{-(1+\beta)} \log \left(u_{i, t-1}\right)+\varepsilon_{i t}
$$

En este caso, si existe convergencia en la evolución de los niveles de desempleo el coeficiente $\beta$ debería ser mayor que cero. La convergencia entre las tasas de desempleo implica que el incremento de la tasa de paro tiene una relación negativa con la tasa de desempleo al inicio del periodo. De modo que países con una mayor tasa de paro al inicio del periodo considerado deberían reducir su tasa de desempleo más rápidamente que países con una tasa de paro inicialmente menor, desencadenando, de este modo, un proceso de convergencia o acercamiento (caching up) entre las tasas de desempleo a lago plazo. De forma implícita se presupone que los países convergen hacia tasas de paro reducidas. La convergencia o caching up supone la reducción de las tasas de desempleo y, por tanto, una aproximación a la baja. A largo plazo la economía alcaza un estado estacionario donde el mercado de trabajo permanece en equilibrio y solo existe un cierto nivel de desempleo friccional, de carácter inevitable, debido principalmente al cambio de puesto de trabajo. De forma paralela, si el mercado de trabajo no alcanza el equilibrio a largo plazo se puede establecer que existen problemas estructurales que impiden el ajuste entre la oferta y la demanda laboral.

\subsection{La $\sigma$-convergencia y su aplicación al desempleo.}

El segundo modo de enfocar el estudio de la convergencia es a través de la $\sigma$ convergencia. La $\sigma$ convergencia estudia las variaciones en la dispersión en un grupo de países y establece que existe convergencia cuando dicha dispersión se reduce en el tiempo. En el terreno empírico la $\sigma$ convergencia se estima a través del análisis de la varianza muestral del logaritmo de la renta (Sala-i-Martin, 1994). En el caso del desempleo sería igual: 


$$
\sigma^{2}{ }_{t}=(1 / N) \sum_{i=1}^{N}\left[\log \left(u_{i t}\right)-\mu_{t}\right]^{2}
$$

En la ecuación anterior se representa el cálculo de la varianza sesgada de una distribución, siendo $u_{i t}$ la tasa de paro para cada uno de los países considerados (i) en un periodo de tiempo $(t), N$ es el en número total de países analizados y $\mu t$ es la media muestral del logaritmo de la tasa de desempleo ${ }^{6}$. La $\beta$-convergencia y la $\sigma$ convergencia mantienen una relación común. La existencia de $\beta$-convergencia es una condición necesaria pero no suficiente para la existencia de $\sigma$ convergencia. Por último, establecer que la tasa de desempleo es una medida que se encuentra acotada entre 0 y 100 . Aunque resulten claramente improbable, los casos extremos de su variación se corresponden con situaciones donde toda la población activa se encuentra ocupada (en cuyo caso la tasa de desempleo sería 0) o donde toda la población activa se encuentra desempleada (en cuyo caso la tasa de desempleo sería 100). En el caso de que la tasa de desempleo fuera cero no se puede calcular su logaritmo y por consiguiente no se puede conocer cuál es la sigma convergencia. La $\sigma^{-}$ convergencia no es una medida adecuada en aquellos casos donde algún elemento del análisis presente valores iguales a cero. No obstante, dado que el anterior caso resulta claramente improbable, generalmente se podrá calcular la dispersión existente entre las tasas de desempleo, y con ello, determinar si existe convergencia.

\subsection{La convergencia absoluta y la convergencia condicional.}

Durante los ochenta, se desarrollaron una serie de estudios en torno a la existencia de convergencia en términos del crecimiento de la renta y del PIB que demostraban de forma empírica la falta o inexistencia de convergencia en sentido $\sigma$ y $\beta$. Parecía que la idea de convergencia se trataba de una posibilidad teórica que no se producían en la realidad económica o que sólo se producían en raras ocasiones bajo desarrollos teóricos bastante complejos. ¿Suponía esto que no se producía una cierta convergencia entre los países? No, todo lo contrario. A principios de los noventa se inicio una contrarrevolución teórica. Varios autores entre ellos

\footnotetext{
${ }^{6} \mathrm{Al}$ realizar la medición respecto a la media se consigue una medida relativa sobre el acercamiento entre las tasas de desempleo. No obstante, aunque matemáticamente sea igual, subjetivamente puede que no se considere igual alcanzar una cierta convergencia entre tasas de desempleo muy elevadas que entre tasas de desempleo más reducidas. Es por ello que posteriormente se analiza la evolución de la diferencia entre la tasa de desempleo de cada país y la media.
} 
Sala-i-Martin (1990), Barro y Sala-i-Martin (1990, 1991 y 1992) y Mankiw, et al (1992) redefinieron el concepto de convergencia utilizado hasta el momento, definido a partir de entonces como convergencia absoluta y propusieron una nueva forma de medir la convergencia a través de un nuevo concepto conocido como convergencia condicional o relativa ${ }^{7}$.

La diferencia entre la convergencia absoluta y relativa se haya en el hecho de que los países sobre los cuales se realizan los análisis de convergencia compartan o no ciertos parámetros. En el cálculo de la convergencia condicional o relativa es una condición necesaria que los países analizados compartan un mismo estado estacionario. De no ser así, se estaría estimando una convergencia entre países con diferentes horizontes a largo plazo, siendo obvio en este caso, la inexistencia de convergencia. Se introduce en el estudio de la convergencia un condicionamiento de ahí su nombre. Desde la teoría económica, existen dos formas de condicionar los datos para el estudio de la convergencia condicional.

La primera forma consiste en realizar el análisis de la convergencia entre economías parecidas o similares. Se seleccionan a priori aquellos países que pueden contar con un estado estacionario similar. Esta idea desarrollada por Baumol (Baumol et al, 1989 y 1994) se conoce como "clubs de convergencia". En este artículo, el argumento anterior es un supuesto de partida, los países europeos son similares entre sí y se supone que cada vez se asemejan más debido a los criterios de convergencia establecidos en Maastrich y al desarrollo de las Directrices Europeas. En los países europeos sería posible estudiar la existencia de convergencia condicional, dado que todas las naciones europeas supuestamente convergen a un mismo estado estacionario. No obstante, este tipo de estudios sobre la convergencia se realizan sobre todo entre regiones o provincias de un mismo país, donde la similitud es máxima gracias a la libre circulación de los factores productivos. Si al estudio de la convergencia en el desempleo aplicamos los desarrollos de Sala-i-Martín (1994) podemos obtener la siguiente ecuación sobre la convergencia condicional:

$$
\gamma_{i\left(t_{0} ; t_{0}+T\right)}=a-\left\lfloor 1-e^{-\beta T} / T\right\rfloor \log \left(U_{i\left(t_{0} ; t_{0}+T\right)}\right)+\varepsilon_{i\left(t_{0} ; t_{0}+T\right)}
$$

En esta ecuación la tasa de crecimiento de la tasa de desempleo

\footnotetext{
${ }^{7}$ Entre las definiciones de convergencia destacan las ofrecidas por Quah (1993) y Barro y Sala-i-Martin (1992).
} 
$\gamma_{i\left(t_{0} ; t_{0}+T\right)}$ depende de la tasa inicial de desempleo medida en logaritmos $\log \left(U_{i\left(t_{0} ; t_{0}+T\right)}\right)$. Se trata de medir el concepto clásico de $\beta$-convergencia a través de una relación no lineal. En la ecuación anterior, $\beta$ es la velocidad de convergencia de la economía al estado estacionario. El coeficiente de la tasa de desempleo en el origen es una función decreciente de la duración del periodo estimado, pero $\beta$ es independiente del periodo de estimación o del tiempo $(T)$ lo cual resulta muy útil para la estimación a nivel empírico.

Una segunda manera de condicionar los datos es utilizar regresiones múltiples, con un número determinado de variables adicionales que actúan como variables explicativas del estado estacionario. Es decir, si no se comparte un mismo estado estacionario se puede condicionar a través de variables explicativas de modo que sea lo más similar posible. Esto supone, realizar sucesivas regresiones sobre distintas variables hasta encontrar una relación negativa entre las variables estudiadas, en este caso entre el crecimiento de la tasa de desempleo y la tasa de desempleo inicial. Esta metodología se conoce como estimaciones a la Barro y permite establecer cuáles, y en que grado de intensidad, son los condicionantes de la convergencia. De acuerdo con esta segunda forma de condicionar, la convergencia condicional en términos del desempleo se podría estimar a través de la siguiente ecuación:

$$
\gamma_{i t}=a-\beta \log \left(u_{i\left(t_{0} ; t_{0}+T\right)}\right)+\phi X_{i t_{0}-1}+\varepsilon_{i t}
$$

Esta ecuación es similar a las anteriores, sólo que ahora incluye un vector $X_{i t_{0}-1}$ de variables explicativas, la condición impuesta es que $\beta$ debe ser un coeficiente negativo tras introducir las variables predeterminadas $\left(X_{i t_{0}-1}\right)$. Así pues, el contraste sobre la convergencia se realiza utilizando diferentes variables hasta que se logra un $\beta$ negativo. En econometría esta estimación es similar al test de variables excluidas. Si queremos estimar la velocidad de convergencia podemos utilizar una ecuación similar a las utilizadas en el apartado anterior, incluyendo las nuevas variables:

$\gamma_{i\left(t_{0} ; t_{0}-1\right)}=a-\left[1-e^{-\beta T} / T\right] \log \left(U_{i\left(t_{0} ; t_{0}-1\right)}\right)+\phi X_{i t_{0}-1}+\varepsilon_{i\left(t_{0} ; t_{0}-1\right)}$

Hoy en día, el concepto previo de convergencia condicional en términos del crecimiento de la renta es objeto de duras críticas. Las más severas han venido de la mano de la conocida como falacia de Galton (Quah, 1993). La convergencia se mide como la aproximación entre una 
serie de economías a lo largo del tiempo, por tanto, se realiza una comparación respecto a una media dinámica. El punto de comparación cambia a lo largo del tiempo, lo que puede dar lugar a una falacia estadística. La convergencia debe venir de la mano de una aproximación real y no de un falso ajuste alrededor de la media a lo largo del tiempo. Esto obliga a realizar contrastes de robustez sobre las series que en numerosos casos no son superados. Por otra parte, para estudiar la convergencia también es necesario realizar contrastes sobre la existencia de cambios estructurales. La convergencia debe ser analizada a largo plazo donde los cambios recientes en la estructura económica no tengan efectos. En este artículo, dentro de las series del desempleo analizadas no se observa ninguna ruptura estructural importante que no permita desarrollar los estudios sobre la convergencia.

Friedman (1992) también ha criticado duramente el término de $\beta$ convergencia a través de la elección del periodo de referencia. La elección del año de inicio, así como el año de finalización del periodo estudiado, pueden conducir a la existencia de convergencia debido a una cuestión estadística. Tanto el año de partida como el de finalización deben pertenecer a periodos de estabilidad económica. Si, por ejemplo, el año de inicio pertenece a un periodo de crisis y el de finalización a una etapa de intenso crecimiento es posible que obtengamos resultados positivos en torno a la existencia de convergencia aunque de forma inadecuada. En este artículo la convergencia se analiza para el periodo muestral de 1977 al año 2000, ambos años no se caracterizan por la existencia de crisis o elevadas creaciones de empleo. Por tanto, el estudio de la convergencia en dicho periodo no debe verse influido por la elección del ámbito temporal. No obstante, esta crítica puede solventarse siempre y cuando conjuntamente al cálculo de la $\beta$-convergencia se ofrezca una estimación de la $\sigma$ convergencia, de modo que se pueda observar si existe una reducción real de la varianza a lo largo del tiempo y no sólo por la comparación de dos momentos del tiempo. Por último, Friedman también critica la agrupación de países conocida como clubs de convergencia, dado que la inclusión o no de ciertos elementos influye en la existencia o no de convergencia y puede conllevar una cierta subjetividad. Tal como se ha establecido previamente, los países analizados en este artículo son los miembros de la UE-15 por tanto su elección no conlleva ningún tipo de subjetividad dado que su análisis se debe a que forman parte de un área económicamente integrada con características únicas. 


\section{Situación actual de la convergencia en el tema del desempleo.}

En primer lugar, al encarar el estudio de la convergencia en términos del desempleo cabe preguntarse, si existen las condiciones iniciales para que se produzca dicho proceso. En el anexo (gráfico A.1) se recoge la evolución del diferencial entre la evolución del desempleo en cada uno de los estados miembros de la UE y la tasa media de desempleo para el conjunto de la UE-15. Esta información establece si existe una aproximación al resto por parte de cada unos de los países europeos. De la evolución particular de cada país se pueden extraer una serie de pautas de comportamiento comunes:

En términos absolutos, en 1976 los países que se encontraban más alejados de la media eran Irlanda (se encontraba a 4,23 puntos porcentuales por encima de la tasa de desempleo media para el conjunto europeo) y Luxemburgo (a 4,12 puntos porcentuales por debajo). En el año 2000 los países más alejados de la media son España (con una tasa de desempleo 5,64 puntos porcentuales por encima de la media europea) y, de nuevo, Luxemburgo (6,45 puntos porcentuales por debajo de la media).

En la mayoría de países europeos el diferencial con la media se esta reduciendo aunque bajo una intensidad moderada. Este hecho es muy favorable a la existencia de un proceso de convergencia en el desempleo dado que implica un cierto acercamiento. Alemania, Bélgica e Italia son los países que se han mantenido más próximos a la media, mientras que, Irlanda, Holanda, España, Portugal y Grecia son los países de la UE que más se han alejado de la media europea. Aunque, tal como se ha establecido previamente, España ha logrado reducir el nivel de desempleo de forma considerable gracias a la buena actuación de las instituciones laborales (Bentolila y Jimeno, 2003).

Se observa que la reducción del diferencial con la media se realiza principalmente a través de dos caminos, o bien, de una forma continuada y sostenible, o a través de movimientos cíclicos y reducciones drásticas. En Austria, Bélgica, Francia, Alemania, Grecia, y Luxemburgo la reducción del diferencial con Europa se ha realizado a través de una lenta aproximación, de forma sostenida y continuada. En Dinamarca, Finlandia, Irlanda y España la reducción del diferencial con Europa se encuentra marcada por la existencia de grandes movimientos cíclicos.

Las crisis económicas aumentan el diferencial con la media europea en mayor medida que las etapas de expansión económica logran su reducción de ahí la persistencia del desempleo europeo. En la actualidad existe una importante etapa de expansiva que además de 
favorecer la reducción del desempleo a limado considerablemente las diferencias entre los países europeos.

\section{Aplicación de los instrumentos de medida neoclásicos de la convergencia al desempleo.}

En este epígrafe, se aplica al desempleo los instrumentos de medida de la convergencia originarios de teoría neoclásica de crecimiento. Tal como se ha establecido, si se pretende estudiar la existencia de un cierto proceso de convergencia entre las tasas de desempleo de los países europeos desde la teoría neoclásica caben dos formas de aproximación, una a través de la $\beta$-convergencia y otra a través de la $\sigma$-convergencia.

\subsection{Una aproximación a la $\beta$-convergencia.}

Para estimar la convergencia entre las tasas de desempleo, en primer lugar se ha optado por estimar la ecuación más sencilla posible, aquella donde el crecimiento de la tasa de desempleo medido en logaritmos se encuentra relacionada con la tasa de desempleo al inicio del periodo, medidas ambas en logaritmos. Por supuesto, este tipo de estimación se corresponde con la estimación de la convergencia condicional dado que se a pre-seleccionado como caso de estudio los países de la UE15 , naciones que deben compartir a priori un mismo estado estacionario al formar parte de una misma estructura económica. La estimación para los 15 países de la UE durante el periodo 1976-2000 es la siguiente8:

$$
\log \left(u_{i, t}\right)-\log \left(u_{i, t-1}\right)=1,44-0,72 \log \left(u_{i, t-1}\right)
$$

$$
(-3.406)
$$

Esta ecuación muestra que efectivamente existe un cierto proceso de convergencia entre las tasas de desempleo europeas dado que el coeficiente $\beta$ resulta ser negativo. Este hecho implica que, en términos generales, aquellas economías con una tasa de desempleo peor en 1976, es decir superior al resto, han reducido su desempleo de forma más intensa

\footnotetext{
${ }^{8}$ Entre paréntesis aparecen los estadísticos t que demuestra que tanto la constante como el estimador son significativos, bajo una probabilidad del 95 por ciento. $\mathrm{R}^{2}=0.6$. Realizando la misma estimación pero controlando la robustez de los errores también se obtiene resultados significativos aunque bajo una probabilidad del 90 por ciento. En este caso, los estadísticos son 4.02 y -3.02 respectivamente. Este resultado confirma la validez de la estimación, y por tanto, la existencia de un proceso de convergencia entre las tasas de desempleo europeas.
} 
determinando, por tanto, la existencia de un caching up entre las tasas de desempleo europeas a nivel macroeconómico.

El gráfico 1 incluye cuál es la recta de ajuste de la ecuación anterior pudiendo comprobar la posición de cada uno de los países de la UE. La recta correspondiente a dicha regresión resulta ser bastante horizontal debido a que Luxemburgo, Suecia y Grecia se encuentran muy alejados del resto. Si elimináramos estos países la convergencia sería mayor.

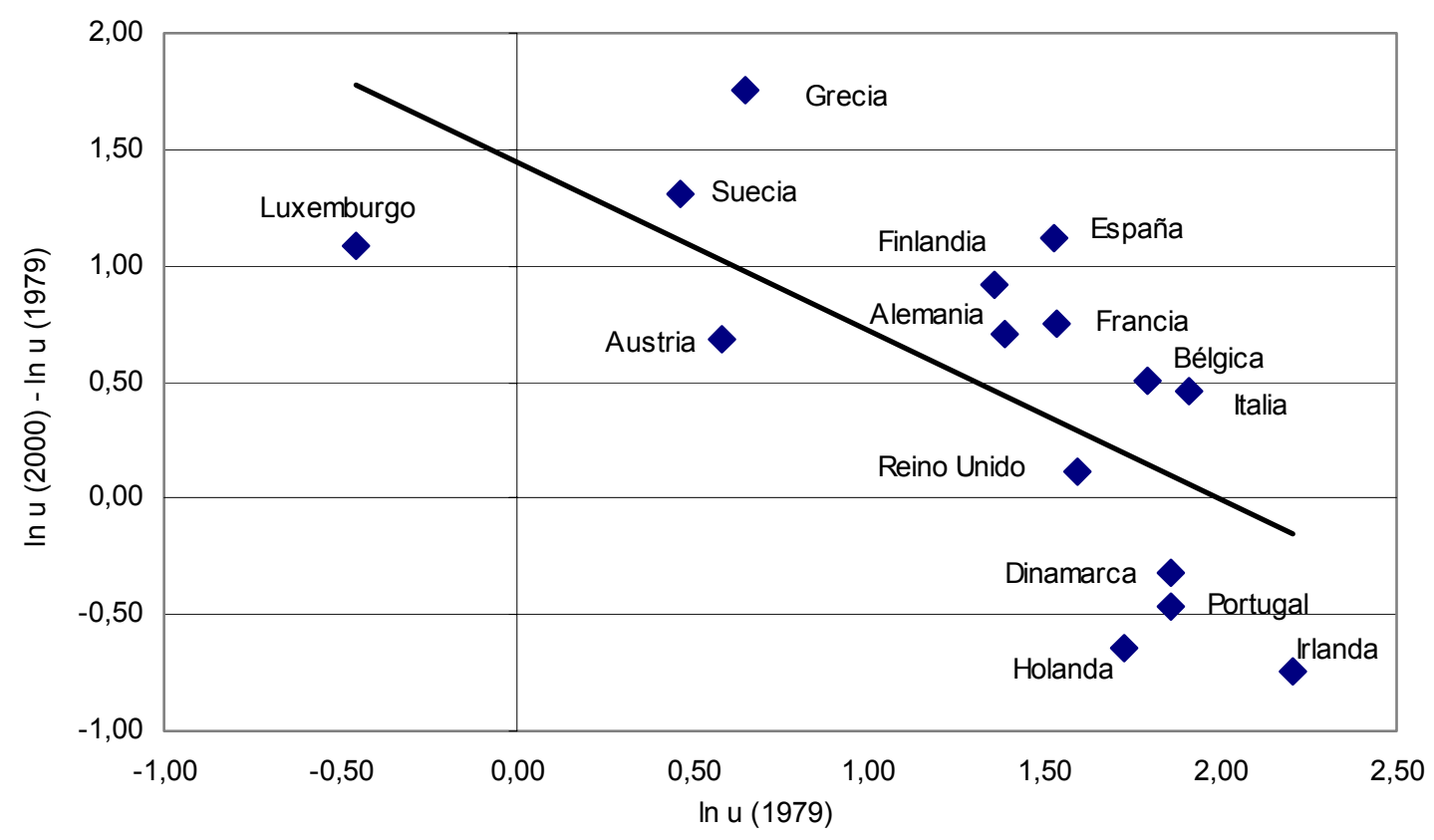

Gráfico 1. Recta de estimación de la convergencia sobre el desempleo. (Fuente: Labour Force Statistics, OCDE).

En el gráfico Luxemburgo se encuentra más alejado del resto porque es el país de la UE que ha mantenido a lo largo del tiempo una menor tasa de desempleo. La posición de Suecia se justifica porque al inicio del periodo, en 1976, su tasa de desempleo era una de las más reducidas de la UE (en torno al 1,5 por ciento). Sin embargo, a raíz de la crisis de los noventa el desempleo en Suecia aumentó considerablemente alcanzando una tasa del 8,2 por ciento en 1993. El reducido nivel de partida refleja sobre todo la existencia en Suecia de un comportamiento totalmente anti-convergente, de ahí su posición en el gráfico. Por último, la 
posición de Grecia se debe sobre todo al intenso crecimiento que ha sufrido su nivel desempleo el cual no ha dejado de aumentar a medida que el país se ha ido desarrollando. El problema principal de Grecia es su alejamiento generalizado de la estructura económica de la UE. Dentro del mercado de trabajo griego existen importantes problemas estructurales tales como la concentración y persistencia del desempleo en determinadas regiones y colectivos.

La ecuación (8) estima la existencia de la convergencia a través de una ecuación lineal simple. Sin embargo, tal como se ha visto en el epígrafe 3 , si se realizan ciertos cálculos aritméticos se puede estimar la convergencia a través de una ecuación exponencial (ecuación 5). En esta nueva estimación, la tasa de crecimiento de la tasa de desempleo, y no el incremento tal y como recogía la ecuación anterior, es la que mantiene una relación directa con la tasa de desempleo al inicio del periodo. Siguiendo la metodología elaborada por Sala-i-Martín (1994) la nueva estimación sería la siguiente ${ }^{9}$ :

$$
\begin{gathered}
\gamma_{t u}=0.06-0.03 \log \left(u_{i, t-1}\right) \\
\beta=0.023 \quad \begin{array}{c}
(-3.406) \\
R^{2}=0.60
\end{array}
\end{gathered}
$$

$\mathrm{Al}$ igual que en el caso precedente, las economías europeas muestran un cierto proceso de convergencia en términos de desempleo dado que el coeficiente de la ecuación (9) es negativo. No obstante, el acercamiento es débil. Para el periodo analizado de 1976 a 2000 se obtiene que la velocidad de convergencia entre las tasas de desempleo europeas es de un 2,3 por ciento anual. Esta estimación resulta mucho más adecuada que la anterior, obteniendo resultados más próximos al proceso que se observa en la UE. Si las economías europeas se aproximaran al ritmo actual y si no existiera ningún tipo de perturbación, bajo una tasa de acercamiento constante del 2,3 por ciento, serían necesarios aproximadamente 50 años para que las tasas de desempleo de los países europeos fueran similares. Por tanto, al igual que sucede en términos de la renta (Sachs y Warner, 1995) la convergencia en el desempleo resulta ser escasa y débil.

$\mathrm{Al}$ realizar los análisis previos sobre la convergencia se presupone que los países europeos comparten un mismo estado estacionario dado que se trata de naciones integrantes de una misma área económica y en algunos casos monetaria. No obstante, tal como se ha explicado

\footnotetext{
${ }^{9}$ De nuevo, controlando la robustez de los residuos se obtiene resultados significativos. Los estadísticos obtenidos en este caso son 4.02 y -3.02 .
} 
anteriormente, aplicando la metodología de Barro se pueden incluir ciertas variables en la ecuación de convergencia para que los países compartan un mismo estado estacionario. Dichas variables solo tendrán sentido y resultaran significativas siempre que favorezcan una mejor medición y estimación del estado estacionario. En este trabajo se han contrastado algunas variables determinantes de la evolución del mercado de trabajo tales como la evolución y el nivel de partida de la población total, la tasas de actividad, la tasa de ocupación, el PIB, el PIB per cápita, el VAB de los servicios y el porcentaje de transferencias sociales sobre el PIB. Sin embargo, tan sólo esta última variable, el porcentaje de transferencias sociales sobre el PIB es determinante en el proceso de convergencia en términos del desempleo. La correlación existente entre la evolución del desempleo y el crecimiento de las transferencias sociales como porcentaje del PIB es del $0,629^{10}$. Si se incluyen las transferencias sociales en la ecuación de convergencia obtenemos una expresión como la siguiente ${ }^{11:}$

$$
\gamma_{t u}=0.03-0.03 \log \left(u_{i, t-1}\right)+0.01 \log \left(T R_{i, t-1}\right)
$$

Las transferencias sociales $\left(T R_{i, t-1}\right)$ contribuyen de forma positiva aunque en una cuantía muy pequeña al proceso de convergencia ${ }^{12}$. La explicación de este hallazgo podría encontrarse en que un mayor crecimiento de las transferencias sociales puede ayudar a reducir el desempleo en aquellos países con una posición de partida peor, lo cual contribuye al proceso de acercamiento entre los niveles de desempleo europeos. Por tanto, a priori, elevar las transferencias sociales en términos del PIB puede ser una adecuada política económica de cara a la reducción del desempleo y a la aproximación al resto de la UE.

\subsection{La $\sigma$-convergencia.}

La $\beta$-convergencia calculada en el apartado anterior se estima sobre la observación de dos momentos del tiempo sin prestar atención al

\footnotetext{
10 Según el índice de correlación de Pearson, a un nivel de significación del 95 por ciento.

11 Entre paréntesis se encuentran los estadísticos de la T-Student. Todos los coeficientes son significativos bajo una probabilidad del 95 por ciento. La ecuación se ha estimado para el periodo 1976-1995 debido a la disponibilidad de los datos.

12 Dicha ecuación ha sido incluida debido a que es la única que obtiene resultados positivos y como ejemplo de la metodología a seguir. No obstante, tal como demuestra el elevado índice de correlación alcanzado, la tasa de desempleo se encuentra muy unida con las transferencias sociales pudiendo originar un elevado proceso de autocorrelación en la ecuación estimada.
} 
proceso intermedio. Por eso es necesario completar el análisis previo con una medida de la convergencia a través de la cual se pueda observar el proceso de convergencia a lo largo de todo el periodo de análisis. La $\sigma$ convergencia es capaz de llevar a cabo este tipo de estudio. La $\sigma$ convergencia establece que existe convergencia cuando la dispersión de una determinada variable dentro de un conjunto de países se ha reducido a lo largo del tiempo. La medida de dispersión que se suele utilizar consiste en el cálculo de una varianza muestral sobre logaritmos tal como se expone a continuación:

$$
\sigma^{2}=(1 / N) \sum_{i=1}^{N}\left[\log \left(u_{i t}\right)-\mu_{t}\right]^{2}
$$

donde $u_{i t}$ es la tasa de desempleo de 1976 a 2000 para cada uno de los países de la UE, $\mu$ t es la media muestral y $N$ el número total de países analizados. El gráfico 2 recoge la estimación de la $\sigma$ convergencia para los países de la UE-15. Para la totalidad del periodo considerado, la dispersión en términos del desempleo se ha reducido levemente, por consiguiente, se puede establecer que ciertamente existe un proceso de convergencia en términos de desempleo a nivel macroeconómico. Las tasas de desempleo europeas se están acercando aunque de forma muy débil ${ }^{13}$.

El crecimiento del empleo producido en la UE en los últimos años, ha favorecido no sólo la reducción del desempleo sino también la reducción de las diferencias entre los países en términos del desempleo. El cálculo de la $\sigma$ convergencia confirma la existencia de un leve proceso de convergencia entre los países europeos en términos de las tasas de desempleo. Recuérdese que la existencia de $\beta$-convergencia es una condición necesaria pero no suficiente para que exista $\sigma$ convergencia. Con el análisis de la $\sigma$ convergencia se ha completado el estudio de la $\beta$ convergencia estableciendo que existe un proceso de acercamiento entre las tasas de desempleo europeas de carácter dinámico a lo largo de todo el periodo considerado.

\footnotetext{
13 Puede observarse que en la etapa de 1984 a 1990 existe un cierto periodo de divergencia. Este hecho se debe al intenso crecimiento del desempleo que se produjo durante estos años en España e Irlanda (Véase el gráfico A.1. del anexo que muestra la diferencia entre la tasa de desempleo de cada uno de los países europeos y la media de la UE-15).
} 


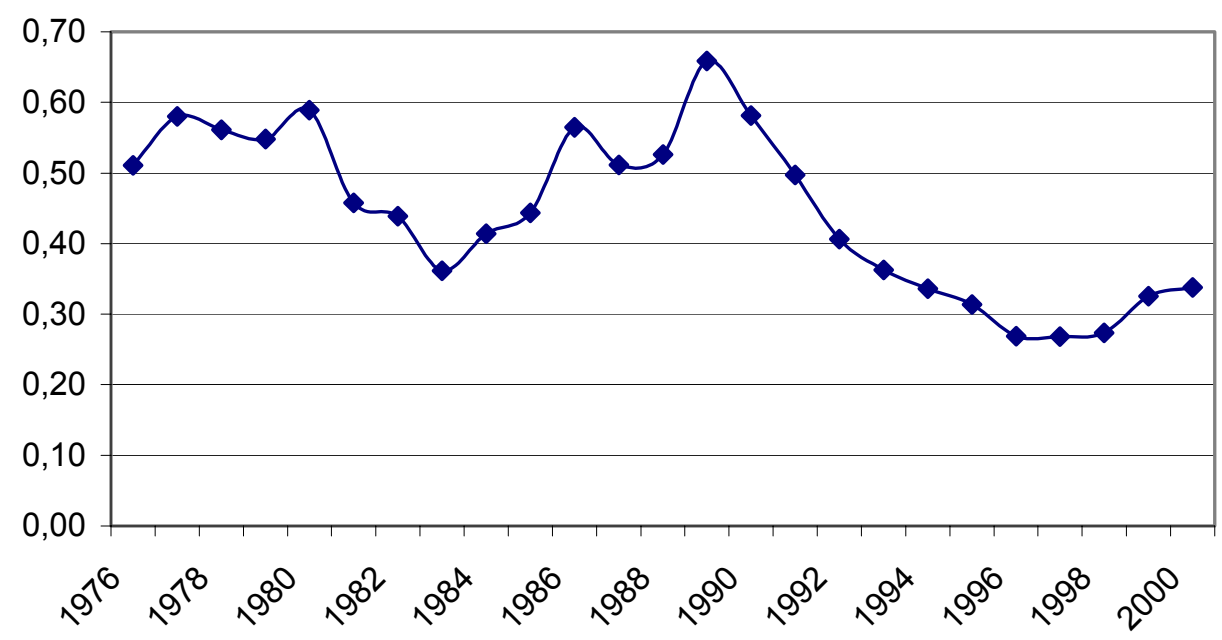

Gráfico 2. La $\sigma$ convergencia o evolución de la dispersión existente entre las tasas de desempleo de los países europeos. (Fuente: Labour Force Statistics, OCDE).

\section{5. ¿Hasta qué punto es firme este proceso?}

Los análisis anteriores muestran que ciertamente existe un proceso de convergencia en términos de desempleo entre las naciones europeas. Sin embargo, la aplicación de los instrumentos de medida neoclásicos al tema del desempleo puede ser insuficiente dado que no muestra hasta que punto dicha relación se trata de algo firme. Por ello el análisis de la convergencia en términos del desempleo se completa con la aplicación del método de Johansen sobre las tasas de desempleo europeas que permite estimar relaciones de cointegración y, por tanto, comprobar la existencia de una convergencia firme en el largo plazo. Tal como se establecía al principio del artículo, se pretende analizar si las tasas de desempleo europeas tienden a un punto común en el largo plazo. La aplicación de la prueba de Johansen exige la existencia de raíces unitarias en las series a tratar. En nuestro caso, se ha comprobado que las tasas de desempleo en primeras diferencias cumplen este requisito ${ }^{14}$. Por tanto, sabemos que podemos aplicar los análisis de cointegración a la evolución

${ }^{14}$ En el anexo se incluyen los test DFA sobre la existencia de raíces unitarias (Cuadro A.1 y A.2). 
de la tasa de desempleo en primeras diferencias entre los países europeos.

Ahora bien, nuestro objetivo es analizar si existe un proceso de convergencia a largo plazo entre los países europeos en términos de desempleo, de modo que, se pueda establecer que existe un acercamiento entre la evolución del desempleo de cada uno de los países de la UE y la evolución general del desempleo para el conjunto de la UE que puede medirse, por ejemplo, a través de la tasa media del desempleo. Sin embargo, este planteamiento conlleva una serie de problemas de autocorrelación; dado que, al estimar la media de los países europeos también se incluye el propio país a estudiar influyendo sobre el resultado total. Por consiguiente, en términos de cointegración no es adecuado estudiar la convergencia de un país respecto a un total del cual forma parte. Este problema se ha solventado estableciendo las relaciones de cointegración entre la tasa de desempleo de cada uno de los países europeos y la tasa media del desempleo para el resto de países europeos excluido el propio país. De este modo se evitan problemas de autocorrelación y también se aproxima una cierta convergencia indirecta a través de la relación con el resto.

El test de Johansen establece las siguientes hipótesis de contraste sobre la posibilidad de cointegración:

1. La primera hipótesis establece que una de las series sea una proporción de la otra en el largo plazo, con lo cual no sería posible la cointegración, dado que no existe una relación real a largo plazo.

2. La segunda hipótesis contrasta la posibilidad de que ambas series sean estacionarias $y$, por tanto, no cointegrables frente a la existencia de al menos un vector de cointegración, es decir, la existencia de una relación válida de cointegración entre ambas variables ${ }^{15}$.

Si se rechazan ambas hipótesis se puede asegurar que ambas series son cointegrables estableciendo la posibilidad de cointegración de las series a través de la siguiente estimación ${ }^{16}$ :

${ }^{15}$ La hipótesis de partida establece la existencia de al menos una relación de cointegración debido a que el contraste se realiza sobre dos series temporales (la tasa de desempleo de un país determinado y la tasa de desempleo media del resto de países, ambas en primeras diferencias).

16 La ventaja de este tipo de test consiste en establecer dos tipos de vectores de corrección del error en función de los incrementos de una u otra variable lo cual mejora el conocimiento sobre la relación de cointegración existente.

$\Delta Y t=\gamma 1(Y t-1-\beta 1+\beta 2 X t-1)+\varepsilon y, t$

$\Delta X t=\gamma 1(Y t-1-\beta 1+\beta 2 X t-1)+\varepsilon x, t$ 


$$
Y_{t}=\rho_{1}+\rho_{2} X_{t}+u_{t}
$$

En el cuadro 1 se han incluido tanto los resultados obtenidos de la aplicación del test de Johansen, como la ecuación de cointegración propuesta por dicho test. En relación con la primera hipótesis sólo se puede rechazar bajo una significatividad del 95 por ciento que la tasa de desempleo nacional no es participe de la tasa de desempleo del resto de países europeos en el caso de Dinamarca, Luxemburgo y España. Para la mayoría de países también se puede rechazar esta proposición pero bajo una significatividad del 90 por ciento. Bélgica, Irlanda y Suecia no cumplen con esta hipótesis y, por tanto, no se puede extraer en estos países la existencia de una relación de cointegración adecuada.

Respecto a la segunda hipótesis los resultados alcanzados también resultan dispares. Con diferente grado de significatividad sabemos que existe al menos un vector de cointegración en el caso de Dinamarca, Francia, Alemania, Grecia, Luxemburgo, Holanda, España y Reino Unido. En estos países se puede establecer una relación a largo plazo entre las tasas de desempleo nacionales y sus correspondientes series del "resto de países". Sin embargo, no se puede establecer lo mismo para los restantes países europeos.

Todos los países con relaciones de cointegración significativas presentan coeficientes dentro de la ecuación de cointegración negativos, lo cual indica la existencia de un proceso de convergencia. En estos países existe una aproximación entre las tasas de desempleo europeas y las tasas de desempleo del resto de países europeos que se mantienen en el largo plazo. En este caso, la convergencia no es una relación casual producida en un momento del tiempo sino que se trata de un proceso firme y duradero. No obstante, el proceso de acercamiento entre las tasas de desempleo resulta parcial y débil. La creación de un mercado de trabajo único similar a todos los países de la UE se encuentra lejos por lo cuál la existencia de un proceso de convergencia entre las tasas desempleo europeas es una cuestión, hoy en día, bastante frágil.

Además, el test de Johansen también permite la incorporación de una constante dentro de la ecuación de cointegración (12), diversos criterios sobre la tendencia a largo plazo y la forma del mecanismo de corrección del error. 
Cuadro 1. Test de Johansen sobre la evolución de la tasa de desempleo en primeras diferencias.

(Fuente: Labour Force Survey, Eurostat 1976-2000).

\begin{tabular}{|c|c|c|c|c|c|c|}
\hline & \multicolumn{2}{|c|}{ HIPÓTESIS 1} & \multicolumn{2}{|c|}{ HIPÓTESIS 2} & \multirow{2}{*}{\multicolumn{2}{|c|}{$\frac{\text { ECUACIÓN }}{\text { Variable dependiente }}$}} \\
\hline & Likelihood & Hypothesized & Likelihood & Hypothesized & & \\
\hline & Ratio (1) & No, of CE(s) & Ratio (2) & No, of CE(s) & $\alpha_{1}$ & T-stat, \\
\hline Austria & n.d. & n.d. & n.d. & n.d. & n.d. & n.d. \\
\hline Bélgica & 11,573 & None & 4,485 & At most 1 & $-1,932$ & $-0,422$ \\
\hline Dinamarca & 18,082 & None ** & 5,829 & At most 1 * & $-0,522$ & $-0,497$ \\
\hline Finlandia & 14,753 & None * & 2,520 & At most 1 & 0,235 & $-0,903$ \\
\hline Francia & 13,038 & None * & 3,909 & At most 1 * & $-1,364$ & $-0,348$ \\
\hline Alemania & 14,587 & None * & 4,225 & At most $1 *$ & $-0,566$ & $-0,236$ \\
\hline Grecia & 14,537 & None * & 6,013 & At most $1 *$ & $-0,929$ & $-0,256$ \\
\hline Irlanda & 10,518 & None & 3,263 & At most 1 & $-0,859$ & $-0,113$ \\
\hline Italia & 15,574 & None * & 3,728 & At most 1 & $-0,929$ & $-0,433$ \\
\hline Luxemburgo & 20,313 & None ** & 6,620 & At most $1 * *$ & $-0,279$ & $-0,051$ \\
\hline Holanda & 14,271 & None * & 6,581 & At most $1 * *$ & $-0,448$ & $-0,402$ \\
\hline Portugal & 14,079 & None * & 3,623 & At most 1 & $-0,974$ & $-0,333$ \\
\hline España & 20,662 & None ** & 4,767 & At most 1 * & $-3,541$ & $-0,483$ \\
\hline Suecia & 10,500 & None & 3,722 & At most 1 & 0,420 & $-0,936$ \\
\hline Reino Unido & 13,680 & None * & 3,910 & At most $1 *$ & $-1,207$ & $-0,255$ \\
\hline
\end{tabular}

Ecuación de contraste: $\Delta$ (Tu Austria; t ) $=\alpha 1 \Delta$ ( Tu RE Austria ; t )

La estimación asume la existencia de una tendencia no determinística en los datos y no incluye constante.

n.d. No disponible debido a la falta de continuidad de la serie estadística.

(1) El valor crítico al 5 por ciento es 12,530 y al 1 por ciento $16,310$.

(2) El valor crítico al 5 por ciento es 3,840 y al 1 por ciento $6,510$.

\section{Conclusiones.}

A lo largo de este artículo se ha establecido que existe un proceso de convergencia entre las tasas de desempleo europeas. Las primeras aproximaciones a esta cuestión nos han mostrado que los países europeos cuentan con una serie de comportamientos muy dispares. La evolución de las tasas de desempleo en cada uno de los países de la UE respecto a la media resulta ser muy heterogénea. No obstante, la mayoría de los países europeos están reduciendo el diferencial que existe entre la tasa de desempleo nacional y la media europea. La falta de homogeneización de las estructuras sectoriales de producción así como las infraestructuras 
públicas (Munnell, 1990) pueden ser los factores determinantes de estas diferencias.

Para demostrar si realmente se produce una convergencia se aplicó al tema del desempleo los instrumentos de medida neoclásicos: la $\beta$ convergencia y la $\sigma$ convergencia. Gracias al primer análisis se ha podido establecer que actualmente existe un proceso de acercamiento entre las tasas de desempleo de los diferentes países europeos. Aquellos países con mayores tasas de desempleo han experimentado reducciones más significativas posibilitando el acercamiento de las economías. Sin embargo, la velocidad de aproximación entre las tasas europeas de desempleo es del 2,3 por ciento, lo cual supone un acercamiento bastante lento.

El cálculo de la $\sigma$ convergencia también confirma la existencia de un proceso de convergencia entre las tasas de desempleo europeas. De forma dinámica se puede establecer que la dispersión entre las tasas de desempleo de los países europeos se está reduciendo aunque, de nuevo, lentamente.

Ambos resultados, de acuerdo con los fundamentos teóricos barajados al principio del artículo, determinan que la movilidad laboral existente en la UE puede que no sea suficiente para equilibrar los mercados de trabajo nacionales, reducir el desempleo y provocar la convergencia. Asimismo, la actuación de las instituciones europeas puede que tampoco resulte determinante cara al logro de la convergencia entre las tasas de desempleo europeas.

Por último, se ha contrastado a través de la aplicación del test de Johansen si el proceso de convergencia alcanzado es firme y se mantiene en el largo plazo. Los resultados obtenidos permiten determinar que el proceso de convergencia es un hecho firme que se produce a largo plazo pero solo para un conjunto de países, es decir, de forma parcial.

En definitiva, desde el ámbito macroeconómico, cabe concluir que existe un proceso de aproximación o acercamiento entre las tasas de desempleo europeas a nivel nacional. No obstante, el proceso de convergencia alcanzado resulta parcial, lento y débil. La escasa convergencia alcanzada puede deberse a la existencia de estructuras sectoriales diferentes, a distintos sistemas en la determinación de los salarios o a la baja movilidad europea, entre otros factores. Todos estos factores deberán estudiarse más adelante para determinar cuál es su influencia sobre el proceso de convergencia y sobre su posible mantenimiento a largo plazo. 


\section{Bibliografía}

Amstrong, H y Taylor J., (2000) "Regional economics and Policy". Blackwell Publishers. Third Edition. USA.

Antolín, P. (1995) "Movilidad laboral, flujos de desempleo, vacantes y comportamiento en la búsqueda de empleo en el mercado de trabajo español". Moneda y Crédito. Na 201.

Barro, R. J. (1997) "Determinants of economic Growth. A cross-country empirical study". The MIT Press. Cambridge, Massachusett. London, England.

Barro, R.J. y Sala-i-Martin, X. (1990) "Economic Growth and Convergence across the United States". NBER Working Paper, 3419.

Barro, R.J. y Sala-i-Martin, X. (1991) "Convergence across states and regions". Brooking Papers on Economic Activity, 1, pp. 107-182.

Barro, R.J. y Sala-i-Martin, X. (1992) “Convergence”. Journal of Political Economy, 100, pp. 2 April. 223-251.

Baumol, W. J., Nelsol, R. y Wolff, E.N. (Editores) (1994) "Convergence of productivity”. Oxford University Press. New York.

Baumol, W. J., Sue Blackman, Anne Batey and Edward N. Wolff (1989) "Productivity and American leadership: The long view". MIT Press, Cambridge MA.

Bayer, C. y Juessen, F. (2004) "Convergence in West German regional Unemployment Rates". Economics Working Paper Archive at WUSTL. Series Urban/Regional. $\quad \mathrm{N}^{\mathrm{o}}$ 0411007. Disponible en: http://econwpa.wustl.edu:80/eps/urb/papers/0411/0411007.pdf

Bernard, A. y Durlauf S. (1996) "Interpreting tests of the convergence hypothesis". Journal of Econometrics, $\mathrm{n}^{\circ}$ 10. pp. 161-173.

Bentolila, S. y Jimeno, J.F. (2003) "Spanish Unemployment: The end of the wild ride?". CEMFI. Working Paper. N 0307. Abril.

Blanchard, O.J. y Katz, L. (1992) "Regional Evolution”, Brooking Paper on economic Activity. 
Comisión Europea (2001) "Employment in Europe 2001. Recent Trends and Prospect". Employment and social affaire. Employment and European Social Funds. http://europa.eu.int.

Decressin, J. y Fatás A. (1995) "Regional Labor Market Dynamics in Europe”. European Economic Review. N 39.

De la Fuente, A. (1996) "Economía regional desde una perspectiva neoclásica. De convergencia y otras historias". Revista de Economía Aplicada N 10 (Vol. IV).

De la Fuente, A. (1998) “Convergencia real? España en la OCDE”. Moneda y Crédito. No 207. pp. 11-57. Madrid.

Friedman, M. (1992) "Do old fallacies ever Die?" Journal of economic Literature. Vol XXX. December. Pp 2129-2132.

Gaulier, G. y Haller, S. (2000) "The Convergence of Automobile Prices in the European Union: an Empirical Analysis for the Period 1993-1999". CEPII research center. Working Papers. N ${ }^{\circ} 2000-14$.

MacConnell, Campbell R. y Brue, Stanley L. (2003) "Economía laboral". Traducción Esther Rabasco. McGraw-Hill, D. L.. Madrid.

Mankiw, N. G., Romer D. y Weil D.N. (1992) "Contribution to the Empiric of Economic Growth”. Quaternary Journal of Economic. N ${ }^{\circ}$ 107-2. Mayo. pp 407-437.

Mulder P. y de Groot H.L.F. (2003) "Sectoral Energy" and LabourProductivity Convergence". Doc. sobre Economía Regional. Banco de la República - Economía Regional. No 000434. Colombia.

Quah, D. (1993) "Galton's fallacy and test of convergence hypothesis", Scandinavian Journal of Economics. Vol, 95, pp. 427-443.

Quah, D. (1995) "Regional convergence clusters across Europe" Centre for Economic Performance. Discussion Paper. N ${ }^{\circ}$ 274. Diciembre.

Ricardo, D. (1817) "Principios de Economía política y Tributación". Editorial. Piramide, 2003. Madrid.

Sachs, J. y Warner, A. M. (1995) "Economic convergence and economic 
Policies" Discussion Paper Number 1715 Febrero. Harvard Institute of economic research. Harvard University. Cambridge. Massachusetts.

Sala-i-Martín, X. (1990) "On growth and states" Tesis doctoral no publicada, Harvard University.

Sala-i-Martín, X. (1994) “Apuntes de crecimiento económico”. Antoni Bosch Editor. Barcelona.

Solow, R.M. (1956) "A contribution to the Theory of Economic Growth". Quarterly Journal of Economics, 70. 1, Feb.

Solow, R.M. (1979) "Another Possible Source of Wage Stickiness" Journal of Macroeconomics. Winter. $\mathrm{N}^{\mathrm{o}} 1$.

Solow, R.M. (1980) “ On Theories of Unemployment” American Economic Review. March n 70.

Solow, R.M. (1992) "El Mercado de trabajo como institución social". Alianza Económica. Madrid.

Van der Hort, A. (2003) "Structural estimate of equilibrium Unemployment in six OECD economies". European Network of economic Policy Research Institutes. Working paper. N ${ }^{\circ}$ 22/july 2003. 
26

Llorente / Revista de Economía Laboral 2 (2005), 1-30

Anexo.

Gráfico A.1. Diferencial con Europa. Puntos porcentuales sobre la media de la tasa de desempleo europeo. 1976-2000.

(Fuente: Labour Force Statistic, OECD.
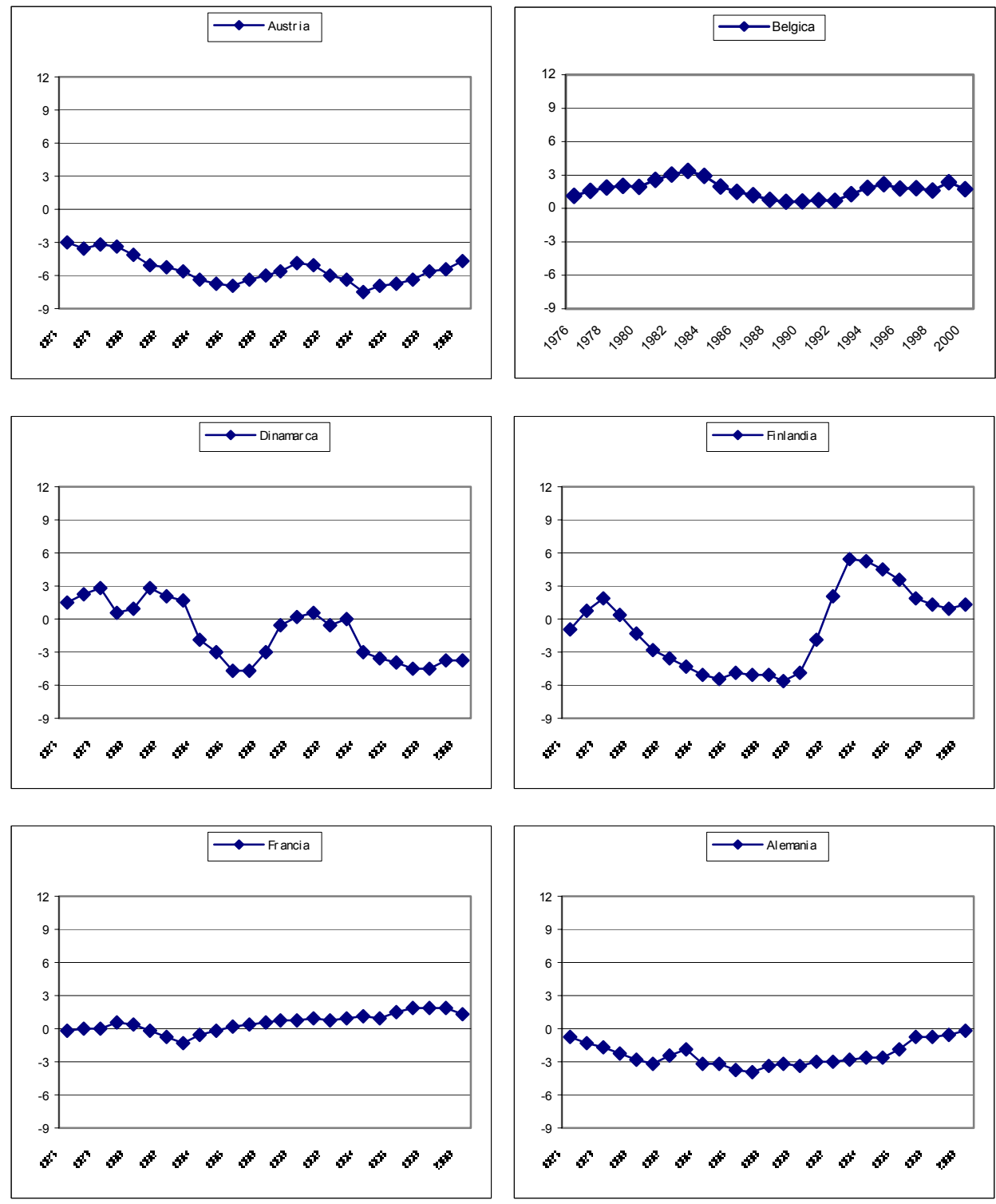
La convergencia entre las tasas de desempleo europeas

27
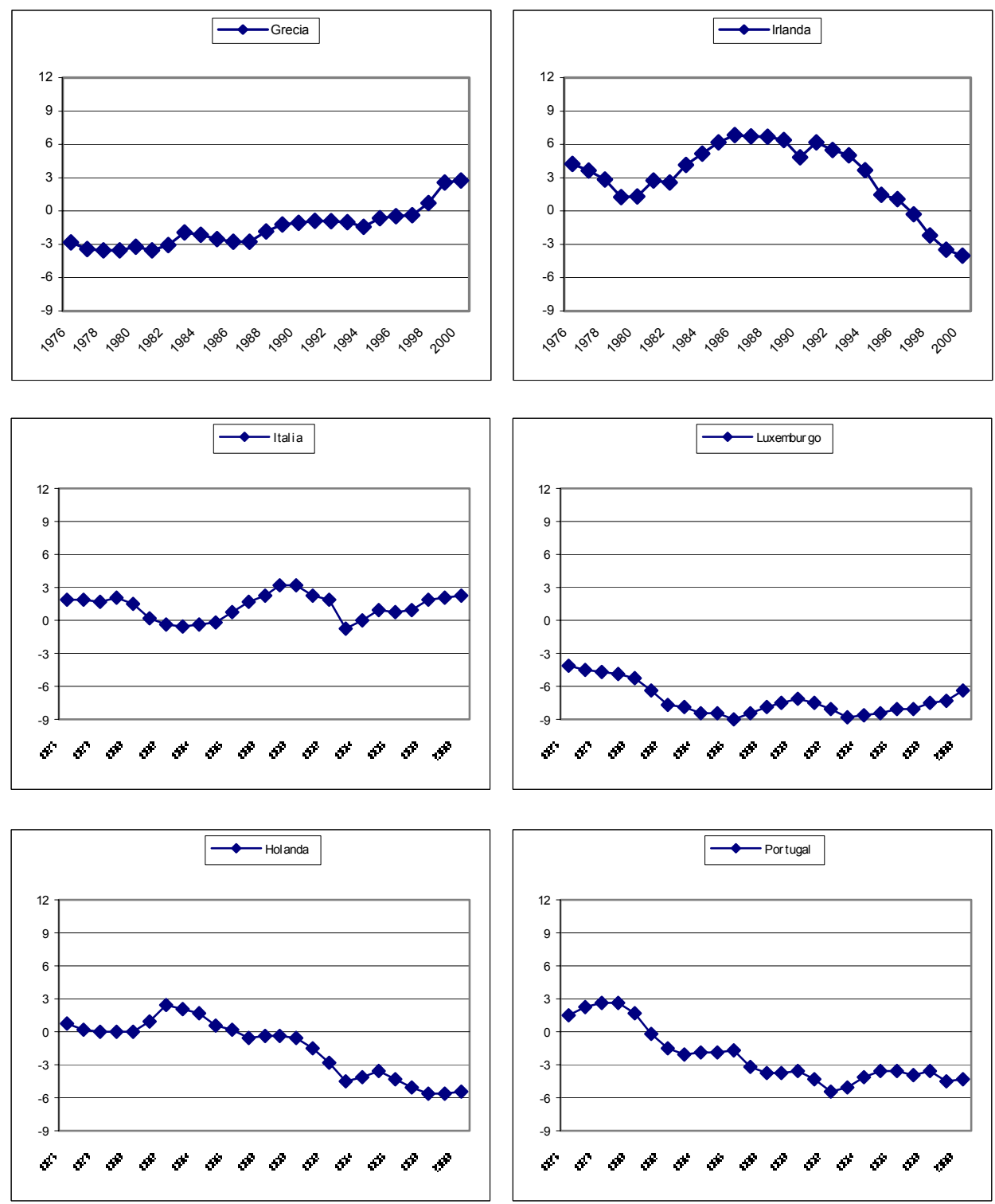
28

Llorente / Revista de Economía Laboral 2 (2005), 1-30
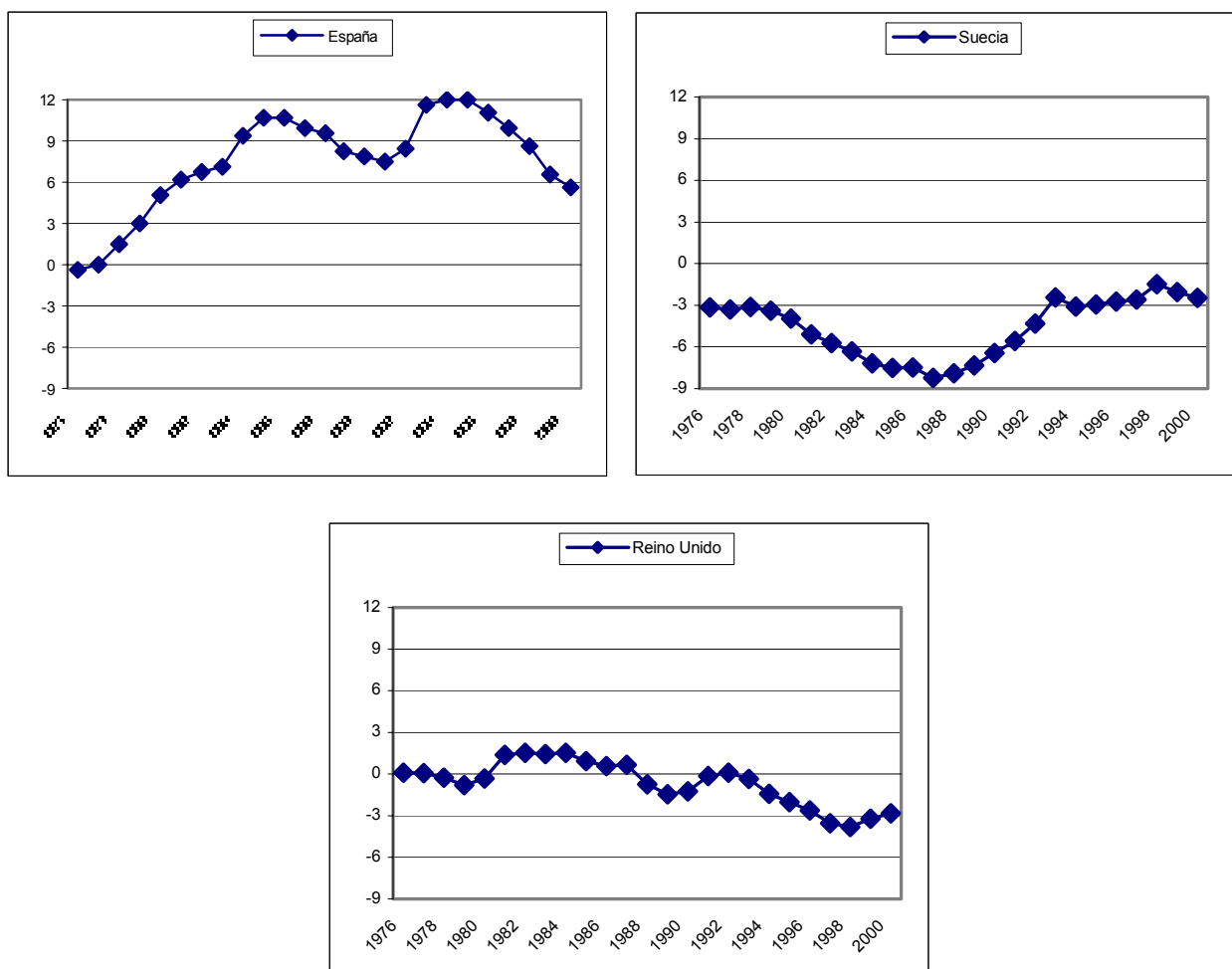
Cuadro A.1. Test DFA sobre las tasa de desempleo europeas. (Fuente: Labour Force Survey, Eurostat 1976-2000).

\begin{tabular}{|c|c|c|c|c|c|}
\hline Países & TEST & SIG. & COEFICIENTE & T-STAT. & SIG. \\
\hline Austria & $-4,247$ & $0,000^{*}$ & $-0,904$ & $-4,247$ & 0,000 \\
\hline Bélgica & $-1,822$ & $0,066^{* * * *}$ & $-0,310$ & $-1,822$ & 0,082 \\
\hline Dinamarca & $-4,029$ & $0,000^{*}$ & $-0,848$ & $-4,029$ & 0,001 \\
\hline Finlandia & $-3,713$ & $0,001^{*}$ & $-0,540$ & $-3,713$ & 0,001 \\
\hline Francia & $-2,094$ & $0,037^{* *}$ & $-0,402$ & $-2,094$ & 0,048 \\
\hline Alemania & $-2,783$ & $0,008^{*}$ & $-0,531$ & $-2,783$ & 0,011 \\
\hline Grecia & $-2,217$ & $0,029^{* *}$ & $-0,378$ & $-2,217$ & 0,037 \\
\hline Irlanda & $-2,497$ & $0,015^{* *}$ & $-0,460$ & $-2,497$ & 0,021 \\
\hline Italia & $-4,019$ & $0,000^{*}$ & $-0,876$ & $-4,019$ & 0,001 \\
\hline Luxemburgo & $-4,519$ & $0,000^{*}$ & $-0,965$ & $-4,519$ & 0,000 \\
\hline Holanda & $-1,991$ & $0,047^{* *}$ & $-0,313$ & $-1,991$ & 0,059 \\
\hline Portugal & $-2,489$ & $0,015^{* *}$ & $-0,405$ & $-2,489$ & 0,021 \\
\hline España & $-2,356$ & $0,021^{* *}$ & $-0,349$ & $-2,356$ & 0,029 \\
\hline Suecia & $-2,331$ & $0,022^{* *}$ & $-0,434$ & $-2,331$ & 0,029 \\
\hline Reino Unido & $-2,456$ & $0,017^{* *}$ & $-0,433$ & $-2,456$ & 0,022 \\
\hline EU-15 & $-2,431$ & $0,018^{* *}$ & $-0,363$ & $-2,431$ & 0,025 \\
\hline EU-15 Media & $-1,845$ & $0,063^{* * * *}$ & $-0,293$ & $-1,845$ & 0,079 \\
\hline \multicolumn{6}{|l|}{ Ecuación de contraste: } \\
\hline \multicolumn{6}{|c|}{$\Delta\left(\mathrm{Tu}_{\text {Austria, } \mathrm{t}}-\mathrm{T} \mathrm{u}_{\text {Austria, } \mathrm{t}-1}\right)=\delta_{1}\left(\mathrm{~T} \mathrm{u}_{\text {Austria, } \mathrm{t}}-\mathrm{T} \mathrm{u}_{\text {Austria } \mathrm{t}-1}\right)+\sum_{\mathrm{i}=1}^{\mathrm{p}-1} \delta_{\mathrm{i}} \Delta\left(\mathrm{Tu} \mathrm{Austria,} \mathrm{t}-\mathrm{T} \mathrm{u}_{\text {Austria } \mathrm{t}-1}\right)$} \\
\hline \multicolumn{2}{|c|}{$\begin{array}{l}* \text { Significativo al } 1 \% \\
* * \text { Significativo al } 5 \% \\
* * * \text { Significativo al } 10 \%\end{array}$} & \multicolumn{2}{|c|}{ Valores de McKinnon: } & \multicolumn{2}{|c|}{$\begin{array}{l}-2.669 \text { al } 1 \% . \\
-1.956 \text { al } 5 \% \\
-1.608 \text { al } 10 \%\end{array}$} \\
\hline
\end{tabular}


Cuadro A.2. Test DFA sobre las tasa de desempleo para el resto de países europeos excepto el país considerado.

(Fuente: Labour Force Survey, Eurostat 1976-2000).

\begin{tabular}{|c|c|c|c|c|c|}
\hline Países & TEST & SIG. & COEFICIENTE & T-STAT. & SIG. \\
\hline Austria & $-1,820$ & $0,066^{* * *}$ & $-0,287$ & $-1,820$ & 0,082 \\
\hline Bélgica & $-1,904$ & $0,056^{* * * *}$ & $-0,306$ & $-1,904$ & 0,070 \\
\hline Dinamarca & $-2,579$ & $0,013^{* *}$ & $-0,390$ & $-2,579$ & 0,018 \\
\hline Finlandia & $-1,714$ & $0,082^{* * * *}$ & $-0,269$ & $-1,714$ & 0,101 \\
\hline Francia & $-1,894$ & $0,057^{* * *}$ & $-0,303$ & $-1,894$ & 0,072 \\
\hline Alemania & $-1,829$ & $0,065^{* * * *}$ & $-0,289$ & $-1,829$ & 0,081 \\
\hline Grecia & $-1,869$ & $0,060^{* * * *}$ & $-0,298$ & $-1,869$ & 0,075 \\
\hline Irlanda & $-2,589$ & $0,012^{* *}$ & $-0,420$ & $-2,589$ & 0,018 \\
\hline Italia & $-1,955$ & $0,050^{* *}$ & $-0,319$ & $-1,955$ & 0,064 \\
\hline Luxemburgo & $-1,829$ & $0,065^{* * * *}$ & $-0,290$ & $-1,829$ & 0,081 \\
\hline Holanda & $-1,960$ & $0,050^{* * *}$ & $-0,322$ & $-1,960$ & 0,063 \\
\hline Portugal & $-1,894$ & $0,057^{* * *}$ & $-0,307$ & $-1,894$ & 0,071 \\
\hline España & $-2,035$ & $0,042^{* *}$ & $-0,343$ & $-2,035$ & 0,054 \\
\hline Suecia & $-1,848$ & $0,063^{* * * *}$ & $-0,291$ & $-1,848$ & 0,078 \\
\hline Reino Unido & $-1,815$ & $0,067^{* * *}$ & $-0,289$ & $-1,815$ & 0,083 \\
\hline EU-15 & $-1,820$ & $0,066^{* * * *}$ & $-0,287$ & $-1,820$ & 0,082 \\
\hline EU-15 Media & $-1,904$ & $0,056^{* * *}$ & $-0,306$ & $-1,904$ & 0,070 \\
\hline \multicolumn{6}{|c|}{ Ecuación de contraste: } \\
\hline \multicolumn{6}{|c|}{ 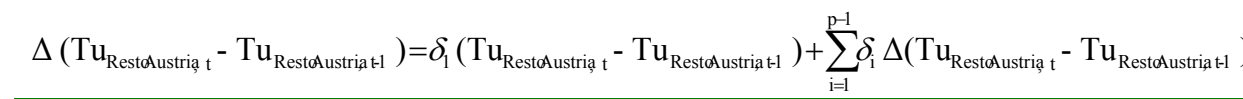 } \\
\hline \multicolumn{2}{|c|}{$\begin{array}{l}* \text { Significativo al } 1 \% . \\
* * \text { Significativo al } 5 \% . \\
* * * \text { Significativo al } 10 \%\end{array}$} & & Valores de McKinno & \multicolumn{2}{|c|}{$\begin{array}{l}-2,669 \text { al } 1 \% \\
-1,956 \text { al } 5 \% \\
-1,608 \text { al } 10 \%\end{array}$} \\
\hline
\end{tabular}

